\title{
Análise polínica e compostos fenólicos de mel e própolis do Pantanal, Mato Grosso, Brasil
}

O objetivo dessa pesquisa foi identificar os tipos polínicos e o teor de compostos fenólicos totais no mel e na própolis do Pantanal, Mato Grosso, Brasil. A pesquisa foi realizada nos municípios de Cáceres, Poconé e Nossa Senhora do Livramento, Mato Grosso. O mel e a própolis foram coletados entre 2011 e 2012 em apiários comerciais, um total de 11 amostras foram coletadas durante o estudo, compondo 6 amostras de mel ( 2 amostras em cada município) e 5 amostras de própolis coletadas no município de Cáceres. A origem botânica das amostras foi por meio da análise polínica das lâminas de mel e própolis. 0 teor de fenólicos totais foi pelo método de Folin-Ciocalteau e flavonoides por cloreto de alumínio. A análise polínica indicou que o mel do Pantanal produzido em Cáceres foi de Myracrodroun. urundeuva e o mel de Poconé e Nossa Senhora do Livramento foi de Mimosa pudica. A própolis do Pantanal não apresentou polens dominantes, os polens acessórios foram Mimosa pudica, Myracrodroun urundeuva e Cecropia sp. Os fenólicos totais no mel foi de 14 a 122,5 mg EAG/g e na própolis foi de 37,97 a 67,42 mg EAG/g. Os tipos polínicos Mimosa pudica, Myracrodroun urundeuva e Cecropia sp. foram recursos importantes para os produtos apícolas no Pantanal de Mato Grosso com teores de fenólicos expressivos.

\section{Pollen analysis and phenolic compounds of honey and propolis from Pantanal, Mato Grosso, Brazil}

\begin{abstract}
The aim of this research was to identify the pollen types and total phenolic compounds content in honey and propolis from Pantanal, Mato Grosso, Brazil. The research was carried out in the municipalities of Cáceres, Poconé and Nossa Senhora do Livramento, Mato Grosso. Honey and propolis were collected between 2011 and 2012 in commercial apiaries, a total of 11 samples were collected during the study, composing 6 samples of honey ( 2 samples in each municipality) and 5 samples of propolis collected in the city of Cáceres. The botanical origin of samples was through pollen analysis of honey and propolis slides. The total phenolic content was by Folin-Ciocalteau method and flavonoids by aluminum chloride. Pollen analysis indicated that Pantanal honey produced in Cáceres was from Myracrodroun urundeuva and honey from Poconé and Nossa Senhora do Livramento was of Mimosa pudica. The Pantanal propolis did not present dominant pollen, the accessory pollen was Mimosa pudica, Myracrodroun urundeuva and Cecropia sp. The total phenolics in honey was 14 to 122.5 mg EAG / g and in propolis was 37.97 to $67.42 \mathrm{mg}$ EAG / g. The pollen types Mimosa pudica, Myracrodroun urundeuva and Cecropia sp. were important resources for bee products from Pantanal of Mato Grosso with expressive phenolic contents.
\end{abstract}

Keywords: Botanic Origin; Apis Mellifera; Honey; Propolis; Pollen.

Gabriela Maria Franz

Universidade Federal de Mato Grosso, Brasil http://lattes.cnpq.br/8309193920543284 gabrielamfranz@gmail.com

Jennifer Obeger Ferreira

Universidade Federal de Mato Grosso, Brasil oberger23@hotmail.com

\section{Loana Longo}

Universidade do Estado de Mato Grosso, Brasil http://lattes.cnpq.br/2827800093113082 loanap 7@hotmail.com

\section{Elaine Maria Loureiro}

Universidade do Estado de Mato Grosso, Brasil http://lattes.cnpq.br/0776360612133074 elayneloureiro@bol.com.br

Joziane da Cruz Mendonça

Universidade do Estado de Mato Grosso, Brasi http://lattes.cnpq.br/0776360612133074 jozi.7@hotmail.com

\section{Kleber Garcia Bárbara}

Universidade do Estado de Mato Grosso, Brasil http://lattes.cnpq.br/6182353358544104 klebergarcia2009@hotmail.com

\section{Carla Galbiati}

Universidade do Estado de Mato Grosso, Brasil http://lattes.cnpq.br/4449909695203792 carla@unemat.br

\section{Referencing this:}

FRANZ, G. M.; FERREIRA, J. O.; LONGO L.; LOUREIRO, E. M.; MENDONÇA J. C.; BÁRBARA, K. G.; GALBIATI, C.. Análise polínica e compostos fenólicos de mel e própolis do pantanal, Mato Grosso, Brasil. Revista Ibero-Americana de Ciências Ambientais, v.9, n.1, p.13-25, 2018. DOI: http://doi.org/10.6008/SPC21796858.2018 .001 .0002 


\section{INTRODUÇÃO}

A apicultura consiste em uma atividade econômica da exploração dos produtos apícolas de Apis mellifera L. Essa atividade tem baixo impacto ambiental e contribui para a conservação da biodiversidade e com o serviço da polinização das plantas cultivadas e silvestres (BALBINO et al., 2015). A apicultura pode ser desenvolvida em todo o espaço geográfico que possui um clima favorável, uma vegetação exuberante e rica em floradas, com rentabilidade e sustentabilidade (SANTOS et al., 2009). A apicultura depende de floradas, água limpa e não tolera queimada, usa a biodiversidade como o pasto apícola, resultando na combinação de geração de renda e conservação ambiental (BRASIL, 2008).

Os produtos apícolas obtidos a partir de floradas silvestres estão cada vez mais escassos, tanto no Brasil como no mundo, em função da redução significativa das áreas com cobertura vegetal nativa (SANTOS e RIBEIRO, 2009). O desenvolvimento da apicultura está se baseando no aproveitamento das culturas florestais e agrícolas, mas, alguns apicultores exploram apenas a vegetação nativa, não alteram o ambiente e inserem mudas das espécies nativas para aumentar as floradas (BALBINO et al., 2015; SANTOS e RIBEIRO, 2009).

Os produtos apícolas são mel, própolis, pólen, geleia real e apitoxina, o mel é o mais conhecido e explorado pelo homem, tanto para uso na alimentação como na medicina tradicional (LIMA, 2005; SANT'ANA et al., 2012; SAXENA et al., 2010). A própolis vem conquistando espaço no campo terapêutico, em razão das suas propriedades biológica (ALMEIDA et al., 2013; BITTENCOURT et al., 2015).

As abelhas usam néctar das flores, composto de frutose e sacarose, saliva e outras substâncias de menor importância para a produção de mel (GOMES et al., 2010, MANZANARES et al., 2011). A própolis é uma resina de coloração e consistência variada, consiste na mistura de substâncias coletadas de partes das plantas, como brotos, botões florais e exsudados resinosos, e as secreções produzidas pelas abelhas (GRAIKOU et al., 2016; GHISALBERTI, 1979).

Assim, o mel e a própolis são dependentes da diversidade florística da região, que representa o conjunto de plantas fornecedoras de pólen, néctar e resina da flora, isso determina a composição química dos produtos apícolas (BARTH et al., 2013, CAN et al., 2015; LOUREIRO e GALBIATI, 2013). A origem botânica do mel e da própolis pode ser determinada pela análise polínica, por meio da identificação dos tipos polínicos das espécies vegetais presentes neles e a quantificação da sua frequência (BARTH, 1989; BARTH et al., 2013).

O mel monofloral foi identificado no Ceará e Piauí com dominância de cipó-uva (Serjania sp) (MORETI et al., 2009; RIBEIRO et al., 2015) e em Tocantins com caju (Anacardium occidentale L.) (ABADIO-FINCO et al., 2010). Em Minas Gerais (Brasilândia de Minas) Simeão et al. (2015) observaram como pólen dominante e Baccharis sp., Copaifera langsdorffii Desf., Eucalyptus spp. e Trema micrantha (L.) Blume.

A análise polínica da própolis indica sua origem geográfica (BASTOS, 2001) e reflete a vegetação e as condições ambientais ao redor do apiário (BARTH, 2006). A descoberta de fontes vegetais na própolis pode auxiliar na caracterização de origem geográfica e botânica e explicação sua ação terapêutica diversa (BANKOVA et al., 2009). 
Os tipos polínicos encontrado nas própolis brasileira são distintos entre regiões geográficas, Souza et al. (2015) encontraram Minosa caesalpiniifolia Benth. como pólen predominante em Barra do Corda (Maranhão), em São Paulo Silva et al. (2013) identificaram 28 tipos diferentes de pólen, entre eles a Acacia sp., Mimosa caesalpiniifolia e Leucaena pallida Britton \& Rose. Na própolis do Nordeste identificou Mimosa verrucosa Benth., Borreria densiflora DC. e Acacia, Mikania e Mimosa pudica L. como dominante (FREITAS et al., 2011, MATOS et al., 2014), no Sul e no Sudeste a Asteraceae e Eucalyptus.

As espécies vegetais contribuem com os compostos secundários, principalmente fenólicos e flavonoides, no mel e na própolis, que são responsáveis pela sua qualidade e atividade biológica (ALQARNI et al., 2012; ALVAREZ-SUAREZ et al., 2010; BITTENCOURT et al., 2015; CHAIKHAM et al., 2016; GÜLÇIN et al., 2010; NEDJI e LOUCIF-AYAD, 2014; SILICI et al., 2010). Os compostos fenólicos são fontes antioxidantes benéficos a saúde humana, que impedem a formação de radicais livres, com potencial protetor contra doenças e danos oxidativos (BANSKOTA et al., 2001; BITTENCOURT et al., 2015; GÜLÇIN et al., 2010; KÜÇÜK et al., 2007; RIBEIRO et al., 2015).

O interesse por produtos naturais com atividade funcional e biológicas cresceu nos últimos anos, o que gerou uma demanda pelos produtos apícolas, devido às suas propriedades nutricionais e terapêuticas (BALTRUŠAITYTĖ et al., 2007; SANT'ANA et al., 2012).

Os fenólicos e flavonoides encontrados no mel brasileiro contribuem com suas propriedades biológicas (RIBEIRO et al., 2015). A própolis verde e vermelha produzida nas regiões brasileiras Sudeste e Nordeste, atualmente são comercializadas por apresentarem propriedades biológicas atribuídas aos compostos fenólicos como artepelin C (BITTENCOURT et al., 2015; CABRAL et al., 2012; GÜLÇIN et al., 2010).

Mato Grosso possui diversidade florística caracterizada em pôr seus três biomas Amazônia, Pantanal e Cerrado (IBGE, 2013), o que pode influenciar na origem e na qualidade do mel e da própolis (OLIVEIRA et al., 2012). O Pantanal ocupa menor área no Mato Grosso, no entanto é bioma ímpar, com maior produção de mel segundo Ferreira et al., (2016), superiores aos outros biomas que compõe a vegetação do Estado.

O mel e a própolis Pantanal de Mato Grosso apresentaram qualidade para o consumo humano, com teor de fenólicos e flavonóides (BASTOS et al., 2011; FRANZ, 2015; LONGO, 2013; LOUREIRO e GALBIATI, 2013), confirmando a importância da biodiversidade local para esses produtos apícolas.

Diante do exposto demonstra-se realizar o interesse de aprofundar estudos sobre a análise polínica e a composição química do mel e da própolis em regiões pantaneiras, pois produtos de origem vegetal distintas podem apresentar diferentes atividades biológicas. Dessa forma, o objetivo dessa pesquisa foi identificar os tipos polínicos e o teor de compostos fenólicos totais no mel e na própolis do Pantanal, Mato Grosso, Brasil.

\section{METODOLOGIA}

\section{Local de estudo}

A pesquisa foi realizada em Mato Grosso, nos municípios de Cáceres, Poconé e Nossa Senhora do 
Livramento, região pertencente ao bioma Pantanal, que ocupa um território de $60,885 \mathrm{~km}^{2}$, no Estado (Tabela 01). A vegetação possui semelhança com o Cerrado, mas apresenta partes de florestas úmidas. A localização do Pantanal em uma depressão geológica favorece inundações sazonais no verão chuvoso, época da enchente (IBGE, 2013). Essa condição de área alagada favorece a existência de ambientes ricos em termos de diversidade, além de uma complexidade paisagística (RADAMBRASIL, 1982).

Tabela 1: Origem geográfica das amostras de mel e própolis do Pantanal Mato Grosso.

\begin{tabular}{clc}
\hline Origem geográfica & \multicolumn{1}{c}{ Coordenadas geográficas } & Safra \\
\hline Cáceres 1 & $16^{\circ} 04^{\prime} 55^{\prime \prime} \mathrm{S} / 57^{\circ} 37^{\prime} 07^{\prime \prime} \mathrm{W}$ & Mel $-2011 / 2012$ \\
Cáceres 2 & $16^{\circ} 00^{\prime} 02^{\prime \prime} \mathrm{S} / 57^{\circ} 39^{\prime} 55^{\prime \prime} \mathrm{W}$ & Própolis - 2011/2012 \\
Cáceres 3 & $15^{\circ} 59^{\prime} 18,7^{\prime \prime} \mathrm{S} / 57^{\circ} 44^{\prime} 21,9^{\prime \prime} \mathrm{W}$ & \\
\hline Poconé 1 & $15^{\circ} 32^{\prime} 54,50^{\prime \prime} \mathrm{S} / 56^{\circ} 32^{\prime} 14,70^{\prime \prime} \mathrm{W}$ & Mel - 2011/2012 \\
Poconé 2 & $16^{\circ} 8^{\prime} 34,10^{\prime \prime} \mathrm{S} / 55^{\circ} 55^{\prime} 42,40^{\prime \prime} \mathrm{W}$ & \\
\hline Nossa Senhora do Livramento 1 & $16^{\circ} 22^{\prime} 20,70^{\prime \prime} \mathrm{S} / 56^{\circ} 37^{\prime} 7,80^{\prime \prime} \mathrm{W}$ & \\
Nossa Senhora do Livramento 2 & $15^{\circ} 41^{\prime} 8,70^{\prime \prime} \mathrm{S} / 56^{\circ} 18^{\prime} 18,90^{\prime \prime} \mathrm{W}$ & \\
\hline
\end{tabular}

\section{Coleta do mel e da própolis}

Os produtos apícolas foram coletados em apiários comerciais. As amostras de mel foram adquiridas junto aos apicultores, provenientes da safra 2011 e 2012, colhidos entre os meses de julho e outubro. As amostras foram armazenadas em frascos âmbar em ambiente climatizado. As amostras de própolis foram obtidas em coletor de própolis inteligente (CPI), raspada do CPI no período de 2011 e 2012, armazenada em sacola plástica atóxica com vedação (tipo zip) e armazenada no freezer.

Um total de 11 amostras foram coletadas durante o estudo, compondo 6 amostras de mel (2 amostras em cada município) e 5 amostras de própolis coletadas no município de Cáceres. A própolis é produzida apenas os apicultores do município de Cáceres no Pantanal de Mato Grosso, demonstrando que estudos de própolis são incipientes (BASTOS et al., 2011; LOUREIRO, 2008; LOUREIRO e GALBIATI, 2013). Cáceres foi o município com maior produção de mel no Pantanal de Mato Grosso em 2011 e 2012 (IBGE, 2013).

\section{Análise polínica do mel e da própolis}

Lâminas de mel foram preparadas a fresco, com gelatina glicerinada pela técnica de Louveaux et al. (1978), conforme o protocolo: $10 \mathrm{~g}$ de mel $10 \mathrm{~mL}$ de água destilada. centrifugada a $3000 \mathrm{rpm}$ por 10 minutos dois tubos. O líquido sobrenadante foi descartado e o sedimento de cada tubo de ensaio foi despejado em duas lâminas; após a secagem do sedimento a lamínula foi fixada com gelatina glicerinada.

As lâminas de própolis foram preparadas de acordo com Barth (1998) e Freitas et al. (2010), com adaptações, para isso a própolis $(0,5 \mathrm{~g})$ foi dissolvidos em álcool e centrifugada a $1500 \mathrm{rpm}$, seguido de fervura em $\mathrm{KOH}$, tratamento por ultrassom e filtragem por tela de 0,3 $\mathrm{mm}$ para eliminação de fragmentos maiores. Em seguida acrescentou a mistura de acetólise (ERDTMAN, 1960), posteriormente levada ao banhomaria por dez minutos, centrifugou novamente a 3000 rpm, descartando o sobrenadante, acrescentou água destilada, centrifugou, acrescentou uma parte de água e uma de glicerina, descansar por 10 minutos, novamente centrifugou e descartou todo o sobrenadante, posteriormente foi retirado o sedimento contido 
no fundo do tubo e confeccionado duas lâminas para identificação dos grãos de polens.

A análise qualitativa foi para identificação dos tipos polínicos, com consulta na palinoteca de referência do Centro de Estudo em Apicultura, Laboratório CETApis - UNEMAT - Campus de Cáceres, bem como comparação com literatura específica (BARTH, 1989; ROUBIK e MORENO, 1991). Quando a identificação não foi possível em nível de família, foi realizado em nível de gênero ou família botânica. A análise quantitativa foi efetuada pela contagem total dos grãos de pólen das lâminas (amostra). A frequência da espécie de pólen foi calculada em função do total de pólen quantificado na lâmina. A frequência dos tipos polínicos foi agrupada em quatro classes de frequência: pólen dominante (PD) é aquele que aparece em mais de $45 \%$ de frequência, pólen acessório (PA) de 45\% a 16\% e pólen isolado (PI) menor que 15\% (BARTH, 1989; LOUVEAUX, 1978). A análise dos dados foi descritiva por meio da média entre as duas lâminas de mel da origem geográfica de cada município, ele foi feito para as lâminas de própolis.

\section{Determinação de compostos fenólicos}

A determinação dos compostos fenólicos foi dividida na quantificação de fenólicos totais e flavonóides as análises laboratoriais foram realizadas no Laboratório do Centro de Estudos em Apicultura (CETApis) na Universidade do Estado de Mato Grosso, Campus Universitário de Cáceres.

\section{Teor de fenólicos totais e flavonoides no mel}

Para a determinação do conteúdo de fenólicos totais foi empregado o método de Folin-Ciocalteau descrito por SINGLETON e ROSSI (1965). Uma alíquota de $100 \mu \mathrm{L}$ de solução aquosa de mel a $0,2 \mathrm{~g} / \mathrm{mL}$ foi misturada a $0,5 \mathrm{~mL}$ de Folin-Ciocalteau e $1,5 \mathrm{~mL}$ de carbonato de sódio $20 \% \mathrm{~m} / \mathrm{v}$, e o volume aferido com água destilada para $10 \mathrm{~mL}$. A leitura foi em absorbância a $765 \mathrm{~nm}$. A quantificação de fenólicos totais foi determinada através da curva padrão de ácido gálico e expressa em mg de equivalentes de ácido gálico gramas de mel (mg de EAG/100g).

O teor de flavonoides foi determinado segundo metodologia descrita por Meda et al. (2005), com adaptações. Uma alíquota de $3 \mathrm{~mL}$ de solução de mel em metanol: água (1:1) na concentração de $500 \mathrm{mg} / \mathrm{mL}$ foi misturada a $3 \mathrm{~mL}$ de solução metanólica a $2 \%$ de cloreto de alumínio hidratado. Após repouso de 30 minutos, as absorbâncias foram lidas no comprimento de onda de $415 \mathrm{~nm}$. A quantificação de flavonoides foi determinada através da curva padrão de quercetina. $O$ conteúdo total de flavonoides foi expresso em mg de equivalentes de quercetina por grama de extrato de mel (mg de EQ/100g).

\section{Teor de fenólicos totais e flavonoides na própolis}

Para determinar o teor de fenólicos totais em extrato de própolis, adicionou-se uma alíquota de extrato de própolis, Folin-Ciocalteau, solução de carbonato de sódio e água destilada. A leitura foi realizada em espectrofotômetro a $760 \mathrm{~nm}$. A quantificação de fenólicos totais foi determinada através da curva padrão de ácido gálico. $\mathrm{O}$ conteúdo total de fenólicos foi expresso em mg equivalente de ácido gálico por grama de extrato de própolis (mg de EAG/100g) (NEDJI e LOUCIF-AYAD, 2014; WOISKY e SALATINO, 1998). 
Para análise de quantificação de flavonoides em extrato de própolis foi adicionada uma alíquota do extrato de própolis, solução de nitrato de alumínio e solução de acetato de potássio e etanol 80 \%. A leitura foi realizada em espectrofotômetro a $415 \mathrm{~nm}$. A quantificação de flavonoides foi determinada através da curva padrão de quercetina. O conteúdo total de flavonoides foi expresso em mg de equivalentes de quercetina por grama de extrato de própolis (mg de EQ/100g) (KOO, 1996; NEDJI e LOUCIF-AYAD, 2014).

\section{RESULTADOS E DISCUSSÃO}

Na análise polínica do mel foram encontrados 41 espécimes, pertencendo a 19 famílias, as com maior frequência Fabaceae com 10 tipos polínicos, Myrtaceae com quatro tipos polínicos e Asteracea, Malvaceae, Sapindaceae e Poaceae com três tipos polínicos cada (Tabela 2).

O mel do Pantanal teve como polens dominantes Myracrodruon urundeuva e Mimosa pudica e como polens acessórios Astronium fraxinifolium, Cecropia sp., Mimosa pudica e Myracrodroun urundeuva (Tabela 2). Essas espécies estiveram presentes em todas as amostras demostrando sua importância para produção de mel no Pantanal, em algumas amostras como polens isolados.

O mel do Pantanal produzido em Cáceres foi de Myracrodruon urundeuva e o mel de Poconé e Nossa Senhora do Livramento foi de Mimosa pudica.

As plantas apícolas ocorrentes no Pantanal foram maiores do que as encontradas no Pantanal de Mato Grosso, com 162 espécies apícolas (POTT e POTT, 1986). Dentre essas confirmou-se a importâncias das espécies aroeira (Myracrodroun urundeuva), gonçaleiro (Astronium. fraxinifolium), picão (Bidens gardneri), Protium heptaphyllum, Ludwigia, Serjania e Byrsonima, espécies que foram comuns ao presente trabalho.

Os tipos polínicos Myracrodroun urundeuva e Mimosa pudica foram característicos no mel da Baixada Cuiabana, outra região do Pantanal de Mato Grosso (LONGO, 2013). Alguns tipos polínicos encontrados nessa pesquisa foram observados no mel em Brasilândia de Minas, MG, entre eles a Astronium sp., Vernonia sp., Mimosa sp., Hyptis sp., Byrsonima sp., Cecropia sp. (SIMEÃO et al., 2015).

Mimosa pudica esteve presente em todas as amostras da presente pesquisa e mel de Santa Luzia do Paruá, MA (MARQUES et al., 2011). Essa espécie é fornecedora de pequena quantidade de néctar e de muitos grãos de pólen (BARTH, 1989), isso pode explicar a ocorrência desse tipo polínico no mel.

Tabela 2: Frequência dos tipos de polens encontrados nas amostras de mel do Pantanal de Mato Grosso, Brasil.

\begin{tabular}{|c|c|c|c|c|c|c|}
\hline \multirow{3}{*}{ Tipos polínicos } & \multicolumn{6}{|c|}{ Amostras } \\
\hline & \multicolumn{2}{|l|}{ Cáceres } & \multicolumn{2}{|c|}{ Poconé } & \multicolumn{2}{|c|}{$\begin{array}{l}\text { Nossa Senhora } \\
\text { do Livramento }\end{array}$} \\
\hline & 1 & 3 & 1 & 2 & 12 & \\
\hline \multicolumn{7}{|l|}{ Amaranthaceae } \\
\hline Amaranthus & & & 0,06 & 0,86 & & \\
\hline \multicolumn{7}{|l|}{ Anacardiaceae } \\
\hline Astronium fraxinifolium Schott & 7,42 & 30,80 & 2,92 & 4,51 & 10,15 & 9,84 \\
\hline Myracrodruon urundeuva Allemão & 53,91 & 28,78 & 8,27 & 21,31 & 6,07 & 8,56 \\
\hline \multicolumn{7}{|l|}{ Asteraceae } \\
\hline Bidens gardneri Baker & & 0,11 & 0,08 & 0,28 & 0,04 & 0,78 \\
\hline Eupatorium maximilianii Schrad. & & & 0,15 & & & 0,20 \\
\hline Vernonia rubricaulis $\mathrm{H}$ etB. & & & 0,01 & 0,35 & 0,27 & \\
\hline \multicolumn{7}{|l|}{ Burceraceae } \\
\hline Protium heptaphyllum (Aubl.) Marchand & & 1,30 & 0,95 & 0,13 & & \\
\hline \multicolumn{7}{|l|}{ Combretaceae } \\
\hline Tipo Combretaceae & & & 0,09 & 0,07 & & \\
\hline
\end{tabular}


Convolvulaceae

Merremia cissoides (Lam.) Hallier $\mathrm{f}$.

0,12

Cyperaceae

Cyperus sp.

Fabaceae

Acacia paniculata Willd.

Anadenanthera colubrina (Vell.) Brenan

Mimosa debilis Humb. \& Bonpl. ex Willd.

Mimosa verrucosa Benth.

Mimosa pudica $\mathrm{L}$.

Senna alata (L.) Roxb.

Senna obtusifolia (L.) H.S. Irwin \& Barneby

Inga sp.

Senna occidentalis (L.) Link

Tipo Fabaceae

Lamiaceae

Hyptis suaveolens (L.) Poit

Malpighiaceae

Byrsonima

Malvaceae

Pseudobombax

Sida

Triumfetta

Melastomataceae

Marcetia

Myrtaceae

Eugenia

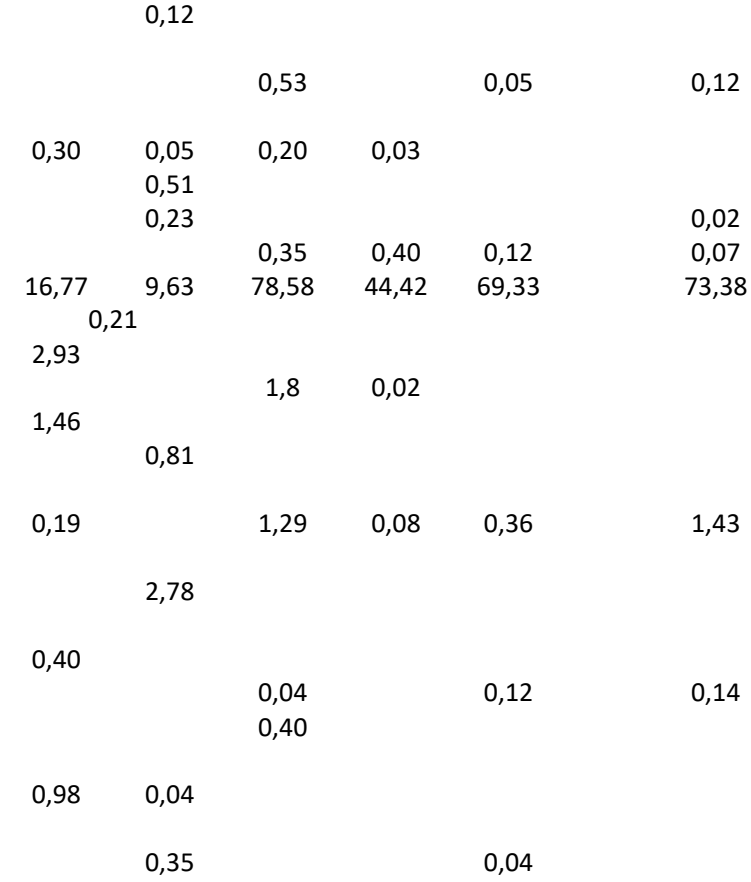

\begin{tabular}{|c|c|c|c|c|c|c|}
\hline Myrcia sp. & & & 0,58 & \multirow{2}{*}{\multicolumn{2}{|c|}{0,07}} & \\
\hline Tipo Myrtaceae & & & & & & \\
\hline Psidium guianense Pers. & & 1,12 & & & & \\
\hline \multicolumn{7}{|l|}{ Onagraceae } \\
\hline Ludwigia tomentosa (Cambess.) H.Hara & 0,01 & 0,05 & & & 0,44 & 0,02 \\
\hline \multicolumn{7}{|l|}{ Poaceae } \\
\hline Brachiaria decumbens Stapf & & 2,17 & & & & \\
\hline Brachiaria sp. & & 1,21 & 0,85 & 1,09 & 2,93 & 2,87 \\
\hline Tipo Poaceae & & & 0,28 & & & \\
\hline Tipo Rubiaceae & & & 0,50 & & & \\
\hline \multicolumn{7}{|l|}{ Sapindaceae } \\
\hline Serjania caracasana (Jacq.) Willd. & & 1,37 & & & & \\
\hline Tipo Serjania & & 12,57 & & & & \\
\hline \multicolumn{7}{|l|}{ Vochysiaceae } \\
\hline Vochysia & & & 0,86 & 0,50 & 0,34 & \\
\hline \multicolumn{7}{|l|}{ Urticaceae } \\
\hline Cecropia sp. & 15,56 & 5,60 & 1,08 & 25,80 & 9,55 & 2,59 \\
\hline Não identificado & & 0,21 & 0,17 & 0,08 & & \\
\hline
\end{tabular}

$\mathrm{Na}$ análise polínica própolis foram observados 38 espécimes, pertencendo a 19 famílias, com maior frequência de Fabaceae (nove tipos polínicos), Rubiaceae (cinco tipos polínicos), Asteracea e Myrtaceae (três tipos polínicos) (Tabela 3).

A própolis do Pantanal não apresentou polens dominantes (Tabela 3), apenas polens acessórios de Mimosa pudica (23,76\%), Myracrodruon urundeuva (27,66\%), Cecropia sp. (17,88 e $30,14 \%)$ e Tipo Anacardiaceae $(29,25 \%)$.

Mimosa pudica foi observada como pólen acessório na amostra de Cáceres 2. Essa espécie esteve presente como pólens dominante e acessório em amostras de própolis de outras regiões brasileiras (FREITAS et al., 2011; MATOS et al., 2014). Essa espécie é de hábito herbáceo, subarbusto nativa, de área antrópica, invasora comum distribuída geograficamente em todas as regiões brasileiras (Flora do Brasil).

O Myracrodroun urundeuva foi um tipo polínico ainda não foi registrado na literatura como pólen presente na própolis brasileira, ele teve presente como pólen acessório na amostra de Cáceres 2. Essa espécie é arbórea, nativa, de área antrópica, ocorrendo no Cerrado, Mata Atlântica e Caatinga (Flora do Brasil), usada 
como recurso para mel em outras regiões do Brasil.

A Cecropia sp. foi observada como pólen acessório na amostra de própolis de Cáceres 1, é uma espécie encontrada como polens acessório e isolado nas amostras de própolis brasileira (FREITAS et al., 2010, 2011; MATOS et al., 2014; SILVA et al., 2013). Ela é espécie arbórea nativa, de área antrópica, ocorrendo no Pantanal Cerrado, Amazonas, Caatinga e Bosque Atlântico (Flora do Brasil), comum no mel brasileiro.

A diversidade de fontes vegetais participam da produção de própolis (BARTH et al., 2013, SILVA et al., 2013, SOUZA et al., 2015), porém a análise polínica não confirma a origem botânica da própolis, apenas a sua origem geográfica e a diversidade vegetal existente no entorno dos apiários.

A análise polínica nas amostras de própolis demostrou que as abelhas utilizam espécies comuns em várias regiões brasileiras com domínio fitogeográfico dos biomas Cerrado, Pantanal, Amazônia, Caatinga e Mata Atlântica, exceto o Myracrodroum urundeuva. Isso reafirma que a análise polínica é uma característica regional que indica o local de produção da própolis.

Tabela 3: Frequência de tipos polens encontrados nas amostras de própolis do Pantanal de Mato Grosso, Brasil.

\begin{tabular}{|c|c|c|c|}
\hline \multirow{3}{*}{ Tipos Polínicos } & \multicolumn{3}{|c|}{ Amostras } \\
\hline & \multicolumn{3}{|c|}{ Cáceres } \\
\hline & 1 & 2 & 3 \\
\hline \multicolumn{4}{|l|}{ Amaranthaceae } \\
\hline Alternanthera tenella Colla & 0,37 & & \\
\hline \multicolumn{4}{|l|}{ Anacardiaceae } \\
\hline Myracrodruon urundeuva Allemão & 6,27 & 27,66 & 3,95 \\
\hline Tipo Anacardiaceae & 6,97 & 1,8 & 29,25 \\
\hline \multicolumn{4}{|l|}{ Apocynaceae } \\
\hline Aspidosperma & 3,23 & & \\
\hline \multicolumn{4}{|l|}{ Asteraceae } \\
\hline Bidens gardneri Baker & 1,40 & 1,08 & \\
\hline Eupatorium maximilianii Schrad. & 0,34 & & \\
\hline Vernonia rubricaulis $\mathrm{H}$ etB. & 0,60 & 1,24 & \\
\hline \multicolumn{4}{|l|}{ Burceraceae } \\
\hline Protium heptaphyllum (Aubl.) Marchand & 4,32 & 0,25 & 2,57 \\
\hline Trema micrantha (L.) Blume & & 0,68 & \\
\hline \multicolumn{4}{|l|}{ Convolvulaceae } \\
\hline Merremia cissoides (Lam.) Hallier $\mathrm{f}$. & 0,21 & 0,08 & \\
\hline \multicolumn{4}{|l|}{ Fabaceae } \\
\hline Acacia paniculata Willd. & 10,32 & 2,72 & 3,85 \\
\hline Bauhinia rufa (Bong.) Steud. & 0,10 & & \\
\hline Cassia grandes L. f. & 0,05 & & 3,85 \\
\hline Inga sp. & 0,04 & & \\
\hline Mimosa caesalpiniifolia Benth. & 0,48 & 0,62 & \\
\hline Mimosa debilis Humb. \& Bonpl. ex Willd. & 14,68 & 0,75 & 8,99 \\
\hline Mimosa pudica $\mathrm{L}$ & 7,74 & 32,76 & 6,32 \\
\hline Platypodium & 0,86 & 0,26 & \\
\hline Senna alata (L.) Roxb. & 0,11 & & \\
\hline \multicolumn{4}{|l|}{ Lamiaceae } \\
\hline Hyptis suaveolens (L.) Poit & 1,79 & 0,36 & \\
\hline \multicolumn{4}{|l|}{ Malvaceae } \\
\hline Hibiscus furcellatus Desr. & 0,03 & & \\
\hline \multicolumn{4}{|l|}{ Tabela 3. Continua } \\
\hline \multicolumn{4}{|l|}{ Melastomataceae } \\
\hline Miconia prasina (Sw.) DC. & 1,94 & 3,33 & 4,55 \\
\hline \multicolumn{4}{|l|}{ Myrtaceae } \\
\hline Myrcia sp.1 & 1,67 & 1,31 & 2,37 \\
\hline Myrcia sp. 2 & 1,52 & & \\
\hline Tipo Myrtaceae & 0,66 & 3,35 & \\
\hline \multicolumn{4}{|l|}{ Onagraceae } \\
\hline Ludwigia tomentosa (Cambess.) H. Hara & 2,78 & 0,63 & \\
\hline \multicolumn{4}{|l|}{ Poaceae } \\
\hline Brachiaria decumbens Stapf & 0,96 & 1,63 & \\
\hline \multicolumn{4}{|l|}{ Rhamnaceae } \\
\hline Ziziphus & & & 0,20 \\
\hline Rubiaceae & & & \\
\hline
\end{tabular}


Borreria densiflora DC.

Rubiaceae

Ferdinantusa sp.

Genipa

Serjania erecta Radlk.

Spermacoce latifolia Aubl.

Solanaceae

Tipo Solanaceae

Urticaceae

Cecropia sp.

Vochysiaceae

Vochysia haenkeana Mart.

Vochysia sp.

Não Identificado
0,24

2,30

2,02

1,72

1,07

0,32

17,88

1,96

0,71

2,22
4,33

1,03

2,16

5,78

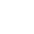

6,21
6,82

0,99

30,14

O mel apresentou teor de fenólicos totais máximo de 122,50 mg EAG/g e mínimo de 14,00 EAG/g. Os flavonoides variaram entre 0,24 a 0,47 mg EQ/g (Tabela 4). O teor de fenólicos totais da presente pesquisa foi semelhante ao encontrados em amostras de méis de Piauí, (31,91 e 123,67 mg de EAG/g) (RIBEIRO et al., 2015). Meda et al. (2005), Chaikham et al. (2016) e Alqarni et al. (2012) analisaram mel de diferentes países e de diferentes origens florais e encontraram teores de fenólicos totais variando entre 0,44 a 114,74 mg de EAG/g de mel, inferior ao da presente pesquisa. Isso indica concentração de fenólicos totais expressiva no mel do Pantanal de Mato Grosso.

Teor de flavonoides avaliado na presente pesquisa foi semelhante a amostras de mel brasileiros que apresentaram de 2,98 e 10,46 mg de EQ/g (Bueno-Costa et al. 2016), da Turquia de 0,65 e 8,10 mg de EQ/g de mel (CAN et al., 2015) e da África de 0,17 e 8,35 mg de EQ/g de mel (MEDA et al., 2005).

O maior teor de fenólicos totais foi na amostra de mel produzidos em Poconé (122,50 mg) e para flavonoides em Nossa Senhora do Livramento $(0,47 \mathrm{mg})$. Esse resultado pode ter influência das espécies mais frequentes nestes locais como Mimosa pudica e Cecropia sp. As espécies isoladas presente apenas em Poconé e Nossa Senhora do Livramento podem ter contribuído para o maior teor de fenólicos desse mel. Isso ocorre em algumas espécies vegetais em estresse devido ao pulso de inundação no Pantanal aumentam a produção de compostos secundários, como fenólicos, usados para proteção da planta (BERTOLDI et al. 2012).

As amostras de mel apresentaram teores de fenólicos e flavonoides variando de um local para outro, esse fato também foi demonstrado em outras pesquisas por Bertoldi et al. (2012) e Meda et al. (2005). Segundo Alqarni et al. (2012) e Can et al. (2015) os compostos fenólicos encontrados em amostras de mel podem apresentar qualidade e quantidade diferenciados de acordo com a origem vegetal.

Os fenólicos e flavonoides são compostos secundários encontrados nas espécies vegetais e são as responsáveis pelas as atividades antioxidante, antimicrobiana, antiviral e anticarcinogênica do mel, que contribui com a saúde humana na prevenção de doenças (KÜçÜK et al., 2007). Diante dos resultados podemos afirmar que o mel produzido no Pantanal de Mato Grosso possui teores de fenólicos e flavonoides semelhantes aos méis comercializados mundialmente, apresentando qualidade.

Tabela 4: Quantificação dos teores de fenólicos totais e flavonoides presentes no mel e na própolis do Pantanal Mato Grosso.

\begin{tabular}{cccc}
\hline Origem geográfica & Produto & Fenólicos totais & Flavonoide \\
\hline Cáceres & Mel & $26 \pm 12$ & $0,24 \pm 0$ \\
& Própolis & $61,15 \pm 11,60$ & $11,16 \pm 5,40$ \\
\hline Poconé & Mel & $77,25 \pm 45,25$ & $0,28 \pm 0,02$ \\
\hline
\end{tabular}


Os fenólicos totais nas amostras de própolis variaram de 37,97 a 67,42 mg EGA/g, e os flavonoides variaram de a 0,93 a 16,30 mg EQ/g (Tabela 04). Os resultados indicam que os teores encontrados estão em conformidade com outras própolis, como os fenólicos totais na própolis marrom brasileira $(48,24 \mathrm{mg} \mathrm{EGA} / \mathrm{g})$ e a própolis do Nordeste (14,8 mg EGA/g), assim como a própolis de Portugal (58,61 a 181,31 mg EGA/g) (BITTENCOURT et al., 2015; CABRAL et al., 2012; SILVA et al., 2012). Os flavonoides foram semelhantes as própolis brasileiras (43 mg EQ/g), do Canadá (7,12 mg EQ/g) e da Turquia $(8,15 \mathrm{mg}$ EQ/g) (ALENCAR et al., 2007; COTTICA et al., 2015; GÜLÇIN et al., 2010).

A variação na composição química da própolis ocorre em função de diferentes fatores, tais como, a diversidade vegetal local, o período da coleta e região da coleta (BANKOVA et al., 2009; BARTH et al., 2013; LOUREIRO e GALBIATI, 2013).

Os compostos fenólicos e flavonóides são responsáveis pelas atividades biológicas da própolis que agem em processos fisiológicos do organismo e atuam como agentes antimicrobianos e antioxidantes capazes de prevenir doença, portanto a o mel e a própolis do Pantanal de Mato Grosso são produtos naturais como fontes propícias de ácidos fenólicos e flavonoides, assim como as frutas e legumes (BANSKOTA et al., 2001; BITTENCOURT et al., 2015; GULÇIN et al., 2010).

O resultado de teor de fenólicos totais do mel de Poconé e Nossa Senhora do Livramento merecem estudos aprofundados que relacione a identidade dos fenólicos com as espécies polínicas, fonte dos compostos secundários.

\section{CONCLUSÕES}

O mel e a própolis do Pantanal de Mato Grosso têm diferente origem botânica entre as amostras, com tipos polínicos de Myracrodruon urundeuva e Mimosa pudica, com contribuição de Cecropia sp. O teor de fenólicos totais e flavonoides foram expressivos no mel e na própolis.

\section{REFERÊNCIAS}

ABADIO-FINCO, F. D. B.; MOURA, L. L.; SILVA I. G.. Propriedades físicas e químicas do mel de Apis mellifera $\mathrm{L}$. Ciência e Tecnologia de Alimentos, Campinas, v.30, n.3, p.706-712, 2010. DOI: http://dx.doi.org/10.1590/S0101$\underline{20612010000300022}$

ALENCAR, S. M.; OLDONI, T. L. C.; CASTRO, M. L.; CABRAL, I. S. R.; COSTA-NETO, C. M.; CURY, J. A.; ROSALEN, P. L.; KEGAKI M.. Chemical composition and biological activity of a new type of Brazilian propolis: Red propolis. Journal of Ethnopharmacology, v.113, n.2, p. 278-283, 2007. DOI: https://doi.org/10.1016/j.jep.2007.06.005

ALMEIDA, E. B.; CARDOSO, J. C.; LIMA, A. K.; OLIVEIRA, N. L.; PONTESFILHO, N. T.; LIMA, S. O.; SOUZA, I. C. L.; ALBUQUERQUE-JÚNIOR, R. L. C.. The incorporation of Brazilian propolis into collagen-based dressing films improves dermal burn healing. Journal of Ethnopharmacology, v.147, n.2, p.419-425, 2013. DOI: https://doi.org/10.1016/j.jep.2013.03.031

ALQARNI, A. S.; OWAYSS, A. A.; MAHMOUD, A. A.. Physicochemical characteristics, total phenols and pigments of national and international honeys in Saudi Arabia. Arabian Journal of Chemistry, v.30, p.1-7, 2012. DOI: https://doi.org/10.1016/i.arabjc.2012.11.013

ALVAREZ-SUAREZ, J. M.; TULIPANI, S.; DIA, D.; ESTEVEZ, Y.; ROMANDINI, S.; GIAMPIERI, F.; DAMIANI, E.; ASTOLFI, P.; BOMPADRE, S.; BATTINO, M.. Antioxidant and antimicrobial capacity of several monofloral Cuban honeys and their correlation with color, polyphenol content and other chemical compounds. Food and Chemical Toxicology, v.48, p.2490-2499, 2010. DOI: https://doi.org/10.1016/j.fct.2010.06.021

BALBINO, V. A.; BINOTTO, E. ; SIQUEIRA, E. S.. Apicultura e responsabilidade social: desafios da produção e dificuldades 
em adotar práticas social e ambientalmente responsáveis.

Revista Eletrônica de Administração, Porto Alegre, v.81, n.2, p.348-377, 2015. DOI: http://dx.doi.org/10.1590/1413$\underline{2311.0442013 .44185}$

BALTRUŠAITYTĖ, V.; VENSKUTONIS, P. R.; ČEKSTERYTĖ, V.. Radical scavenging activity of different floral origin honey and beebread phenolic extracts. Food Chemistry, v.101, p.502-514, 2007. DOI: https://doi.org/10.1016/i.foodchem.2006.02.007

BANKOVA, V.. Chemical diversity of propolis makes it a valuable source of new biologically active compounds. Journal of ApiProduct and ApiMedical Science, v.1, n.2, p. 23-28, 2009. DOI: http://dx.doi.org/10.3896/IBRA.4.01.2.01

BANSKOTA, A. H.; TEZUKA, Y.; KADOTA, S.. Recent Progress in Pharmacological Research of Propolis. Phytotherapy Research, v.15, n.7, p. 561-571, 2001. DOI: http://dx.doi.org/10.1002/ptr.1029

BARTH, O. M.. Palynological analysis of geopropolis samples obtained from six species of Meliponinae in the Campus of the Universidade de Ribeirão Preto, USP, Brazil. Apiacta, v.41, n.2, p. 71-85, 2006

BARTH, O. M.; FREITAS, A. S.; MATSUDA, A. H.; ALMEIDAMURADIAN, L. B.. Botanical origin and Artepillin-C content of Brazilian propolis samples. Grana, v.52, n.2, p.129-135, 2013. DOI: https://doi.org/10.1080/00173134.2012.747561

BARTH, O.M.. O pólen no mel brasileiro. Editora Luxor, Rio de Janeiro, 1989.

BASTOS, E. M. A. F.. Origem botânica e indicadores de qualidade da própolis verde produzida no estado de Minas Gerais, Brasil. Tese (Doutorado em Entomologia) Universidade de São Paulo, Ribeirão Preto, 2001. BASTOS, E. M. A. F.; GALBIATI, C.; LOUREIRO, E. M.; SCOARIS D. O.. Indicadores físico-químicos e atividade antibacteriana de própolis marrom frente à Escherichia coli. Arquivo Brasileiro de Medicina Veterinária e Zootecnia, Belo Horizonte, v.63, n.5, p.1255-1259, 2011. DOI: http://dx.doi.org/10.1590/S0102-09352011000500032

BERTOLDI, F. C.; GONZAGA, L. V.; FETT, R.; DOS REIS, V. D. A. Avaliação da atividade antioxidante e determinação de compostos fenólicos totais de méis produzidos no Pantanal. Evidência, Joaçaba, v.12, n.2, p.155-164, 2012.

BITTENCOURT, M. L.; RIBEIRO, P. R.; FRANCO, R. L.; HILHORST, H. W.; DE CASTRO, R. D.; FERNANDEZ, L. G.. Metabolite profiling, antioxidant and antibacterial activities of Brazilian propolis: Use of correlation and multivariate analyses to identify potential bioactive compounds. Food Research International, v.76, n.3, p.449-457, 2015. DOI: https://doi.org/10.1016/i.foodres.2015.07.008

BRASIL. Plano Amazônia Sustentável: diretrizes para o desenvolvimento sustentável da Amazônia Brasileira. Brasília: MMA, 2008

BUENO-COSTA, F. M.; ZAMBIAZI, R. C.; BOHMER, B. W.; CHAVES, F. C.; SILVA, W. P.; ZANUSSO, J. T. ; DUTRA, I.. Antibacterial and antioxidant activity of honeys from the state of Rio Grande do Sul, Brazil. LWT - Food Science and Technology, v.65, p.333-340, 2016. DOI: https://doi.org/10.1016/i.lwt.2015.08.018

CABRAL, I. S. R.; OLDONI, T. L. C.; ALENCAR, S. M.; ROSALEN, P. L.; IKEGAKI, M.. The correlation between the phenolic composition and biological activities of two varieties of Brazilian propolis (G6 and G12). Brazilian Journal of Pharmaceutical Sciences, São Paulo, v.48, p.557-564, 2012. DOI: http://dx.doi.org/10.1590/S1984-82502012000300023

CAN, Z.; YILDIZ, O.; SAHIN, H.; TURUMTAY, E. A.; SILICl, S.; KOLAYLI, S.. An investigation of Turkish honeys: Their physico-chemical properties, antioxidant capacities and phenolic profiles. Food Chemistry, v.180, p.133-141, 2015. DOI: https://doi.org/10.1016/i.foodchem.2015.02.024

CHAIKHAM, P.; KEMSAWASD, V.; APICHARTSRANGKOON, A. Effects of conventional and ultrasound treatments on physicochemical properties and antioxidant capacity of floral honeys from Northern Thailand. Food Bioscience, v.15, p.19-26, 2016. DOI: https://doi.org/10.1016/j.fbio.2016.04.002

COTTICA S. M.; SABIK, H.; ANTOINE, C.; FORTIN, J.; GRAVELINE, N.; VISENTAINER, J. V.; BRITTEN, M.. Characterization of Canadian propolis fractions obtained from two-step sequential extraction. LWT - Food Science and Technology, v.60, p.609-614,2015. DOI: https://doi.org/10.1016/j.Iwt.2014.08.045

ERDTMAN, G. The acetolysis method: a revised description Svensk Botanisk Tidskrift Lund, v.39, p.561-564, 1960.

FERREIRA, J. O.; GALBIATI, C.; NEVES, S. M. A. S.; MIRANDA, M. R. S.. Produção de mel nos biomas Amazônia, Cerrado e Pantanal, em Mato Grosso, no período de 2002 a 2011. Desenvolvimento e Meio Ambiente, Curitiba, v.39, p.159171, 2016. DOI: http://dx.doi.org/10.5380/dma.v39i0.46505

FLORA DO BRASIL. Jardim Botânico, Rio de Janeiro, 2020.

FRANZ, G. M.. Atividade biológica e composição química de méis produzidos em alguns municípios da Amazônia, Cerrado e Pantanal de Mato Grosso. Dissertação (Mestrado em Ciências Ambientais) - Universidade do Estado de Mato Grosso, Cáceres, 2015.

FREITAS, A. S.; BARTH, O. M.; LUZ, C. F. P.. Própolis marrom da vertente atlântica do Estado do Rio de Janeiro, Brasil: uma avaliação palinológica. Revista brasileira Botânica, São Paulo, v.33, n.2, p.343-354, 2010. DOI: http://dx.doi.org/10.1590/S0100-84042010000200015

FREITAS, A. S.; BARTH, O. M.; SALES, E. O.; MATSUDA, A. H.; ALMEIDA-MURADIAN, L. B.. A palynological analysis of Brazilian propolis samples. Journal of ApiProduct and ApiMedical Science, v.3, n.2, 67-74, 2011. DOI: http://dx.doi.org/10.3896/IBRA.4.03.2.01

GHISALBERTI, E. L.. Propolis: A review. Bee World, v.60, p.59-84.1979.

GOMES, S.; DIAS, G. L.; MOREIRA, L. L.; RODRIGUES, P.; ESTEVINHO, L.. Physicochemical, microbiological and antimicrobial properties of commercial honeys from Portugal. Food Chemistry, v.48, p. 544-548, 2010. DOI: https://doi.org/10.1016/j.fct.2009.11.029 
GRAIKOU, K.; POPOVA, M.; GORTZI, O.; BANKOVA, V.; CHINOU, I.. Characterization and biological evaluation of selected Mediterranean própolis samples. Is it a new type? LWT - Food Science and Technology, v.65, p.261-267, 2016. DOI: https://doi.org/10.1016/j.Iwt.2015.08.025

GÜLÇIN, I.; BURSAL, E.; ŞEHITOĞLU, M. H.; BILSEL, M.; GÖREN, A. C.. Polyphenol contents and antioxidant activity of lyophilized aqueous extract of propolis from Erzurum, Turkey. Food and Chemical Toxicology, v.48, n.8, p.22272238, 2010. DOI: https://doi.org/10.1016/j.fct.2010.05.053

IBGE, 2013. Instituto Brasileiro de Geografia e Estatística.

KOO, H.. Estudo dos flavonóides da própolis de Apis mellifera africanizada provenientes de diversas regiões do Brasil. Dissertação (Mestrado em Ciência dos Alimentos) Universidade Estadual de Campinas, Campinas,1996.

KÜÇÜK, M.; KOLAYL, S.; KARAOĞLU, S.; ULUSOY, E.; BALTAC, C.; CANDAN, F.. Biological activities and chemical composition of three honeys of different types from Anatolia. Food Chemistry, v.100, p.526-534, 2007. DOI: https://doi.org/10.1016/j.foodchem.2005.10.010

LIMA, S. A. M.. A apicultura como alternativa social, econômica e ambiental para a XI Mesorregião do Noroeste do Paraná. Dissertação (Mestrado em Engenharia Florestal do Setor de Ciências Agrárias) - Universidade Federal do Paraná, Curitiba, 2005.

LONGO, L.. Caracterização da apicultura na Baixada Cuiabana no Pantanal Matogrossense. Dissertação (Mestrado em Ciências Ambientais) - Universidade do Estado de Mato Grosso, Cáceres, 2013.

LOUREIRO, E. M.; GALBIATI, C.. Evaluation of the influence of seasonality and landscape on the physicochemical characteristics of propolis. Food Science and Technology, Campinas, v.33, n.4, p.790-795, 2013. DOI: http://dx.doi.org/10.1590/S0101-20612013000400027

LOUREIRO, E. M.. Avaliação da qualidade da própolis produzida em Cáceres - MT. Dissertação (Mestrado em Ciências Ambientais) - Universidade do Estado de Mato Grosso, Cáceres, 2008.

LOUVEAUX, J.; MAURIZIO, A.; VORWO, H. L. G.. Methods of melissopalinology. Bee World, v.59, p.139-157, 1978. MANZANARES, A. B.; GARCIA, Z. H.; GALDÓN, B. R.; RODRÍGUEZ, E. R.; ROMERO, C. D.. Differentiation of blossom and honeydew honeys using multivariate analysis on the physicochemical parameters and sugar composition. Food Chemistry, v.126, p.664-672, 2011. DOI: https://doi.org/10.1016/j.foodchem.2010.11.003

MARQUES, L. J. P.; MUNIZ, F. H.; LOPES, G. S.; SILVA, J. M.. Levantamento da flora apícola em Santa Luzia do Paruá, Sudoeste da Amazônia, Maranhão. Acta Botanica Brasilica, Feira de Santana, v.25, n.1, p.141-149, 2011. DOI: http://dx.doi.org/10.1590/S0102-33062011000100017

MATOS, V. R.; ALENCAR, S. M.; SANTOS, F. A. R.. Pollen types and levels of total phenolic compounds in propolis produced by Apis mellifera L. (Apidae) in an area of the Semiarid Region of Bahia, Brazil. Annals of the Brazilian Academy of Sciences, Rio de Janeiro, v.86, n.1, p.407-418, 2014. DOI: http://dx.doi.org/10.1590/0001-376520142013-0109

MEDA, A.; LAMIEN, C. E.; ROMITO, M.; MILLOGO, J.; NACOULMA, O. G.. Determination of the total phenolic, flavonoid and proline contents in Burkina Fasan honey, as well as their radical scavenging activity. Food Chemistry, v.91, p.571-577, 2005. DOI:

https://doi.org/10.1016/j.foodchem.2004.10.006

MORETI, A. C. C. C.; SODRÉ, G. S.; MARCHINI, L. C.; OTSUK, I. P.. Características físico-químicas de amostras de méis de Apis mellifera L. do Estado do Ceará, Brasil. Ciência e Agrotecnologia, Lavras, v.33, n.5, p. 191-199, 2009. DOI: http://dx.doi.org/10.1590/S1413-70542009000100027

NEDJI, N.; LOUCIF-AYAD, W.. Antimicrobial activity of Algerian propolis in foodborne pathogens and its quantitative chemical composition. Asian Pacific Journal of Tropical Medicine, v.4, n.6, p.433-437, 2014. DOI: https://doi.org/10.1016/S2222-1808(14)60601-0

OLIVEIRA, L. J.; RAUSCHKOLB, A. S.; FIGUEIREDO, A. M. R. Transações e governança na apicultura de Mato Grosso $O$ caso da Apisnorte. Revista de Política Agrícola, Brasília, v.21, n.4, p.20-34, 2012

POTT, A.; POTT, V. J.. Inventário da Flora Apícola do Pantanal em Mato Grosso do Sul Corumbá: Embrapa Pantanal, 1986.

RADAMBRASIL. Levantamentos dos Recursos Naturais. Ministério das Minas e Energia. Projeto RADAMBRASIL: Rio de Janeiro, 1982.

RIBEIRO, J. G.; PIRES, P. S. S.; BRANDÃO, T. M.; SILVA, R. A.. Fenólicos totais e atividade antioxidante de méis de abelha de diferentes floradas. Revista Eletrônica Nutritime, v.12, n.1, p.3903-3909, 2015.

ROUBIK, D. W.; MORENO, J. E.. Pollen and spores of Barro Colorado Island. Missouri Botanical Garden, St. Louis. 1991.

SANT'ANA, L.; SOUSA, J.; SALGUEIRO, F.; AFFONSO, M. C.; CASTRO, R.. Characterization of monofloral honeys with multivariate analysis of their chemical profile and antioxidant activity. Journal of Food Science, v.71, p.135140, 2012. DOI: https://doi.org/10.1111/j.17503841.2011.02490.x

SANTOS, C. S.; RIBEIRO, A. S.. Apicultura uma alternativa na busca do desenvolvimento sustentável. Revista verde, Mossoró, v.4, n.3, p.1-6, 2009.

SAXENA, S.; GAUTAM, S.; SHARMA. A.. Physical, biochemical and antioxidant properties of some Indian honeys. Food Chemistry, v.118, p.391-397, 2010. DOI: https://doi.org/10.1016/i.foodchem.2009.05.001

SILICI, S.; SAGDIC O.; EKICI, L.. Total phenolic content, antiradical, antioxidant and antimicrobial activities of Rhododendron honeys. Food Chemistry, v.121, p.238-243, 2010. DOI: https://doi.org/10.1016/j.foodchem.2009.11.078

SILVA, C. R.; PUTAROV, B. T.; ORSI, R. O.. Pollen spectrum of propolis samples from São Paulo State, Brazil. Acta Scientiarum. Animal Sciences, Maringá, v.35, n.3, p.297300, 2013. DOI: 
http://dx.doi.org/10.4025/actascianimsci.v35i3.18389

SILVA, J. C.; RODRIGUES, S.; FEÁS, X.; ESTEVINHO, L. M.. Antimicrobial activity, phenolic profile and role in the inflammation of propolis. Food Chemical Toxicology, v.50, n.5, p.1790-1795, 2012. DOI:

https://doi.org/10.1016/j.fct.2012.02.097

SIMEÃO, C. M. G.; SILVEIRA, F. A.; SAMPAIO, I. B. M.; BASTOS, E. M. A. F.. Pollen analysis of honey and pollen collected by Apis mellifera Linnaeus, 1758 (Hymenoptera, Apidae), in a mixed environment of Eucalyptus plantation and native Cerrado in Southeastern. Brazilian Journal of Biology, São Carlos, v.75, n.4, p.821-829, 2015. DOI: http://dx.doi.org/10.1590/1519-6984.23513
SINGLETON, V. L.; ROSSI, J. A. Jr.. Colorimetry of total phenolics with phosphomolybdic-phosphotungstic acid reagents. American Journal of Enology and Viticulture, v.16, p.144, 1965 .

SOUZA, H. R.; CORRÊA, A. M. S.; CRUZ-BARROS, M. A. V.; ALBUQUERQUE, P. M. C.. Espectro polínico da própolis de Scaptotrigona aff. postica (Hymenoptera, Apidae, Meliponini) em Barra do Corda, MA, Brasil. Acta Amazônica, Manaus, v.45, n.3, p.307-316, 2015. DOI: http://dx.doi.org/10.1590/1809-4392201403663

WOISKY, R.; SALATINO, A.. Analysis of propolis: some parameters and procedures for chemical quality control. Journal of Apicultural Research, v.37, n.2, p.99-102, 1998. DOI: https://doi.org/10.1080/00218839.1998.11100961

A CBPC - Companhia Brasileira de Produção Científica (CNPJ: 11.221.422/0001-03) detém os direitos materiais desta publicação. Os direitos referem-se à publicação do trabalho em qualquer parte do mundo, incluindo os direitos às renovações, expansões e disseminações da contribuição, bem como outros direitos subsidiários. Todos os trabalhos publicados eletronicamente poderão posteriormente ser publicados em coletâneas impressas sob coordenação da Sustenere Publishing, da Companhia Brasileira de Produção Científica e seus parceiros autorizados. Os (as) autores (as) preservam os direitos autorais, mas não têm permissão para a publicação da contribuição em outro meio, impresso ou digital, em português ou em tradução. 